Pesq. Vet. Bras. 37(9):963-970, setembro 2017 DOI: $10.1590 / \mathrm{S} 0100-736 X 2017000900011$

\title{
Administração seriada de salina hipertônica 7,5\% na terapia para sepse grave decorrente da síndrome da diarreia hemorrágica aguda em cães ${ }^{1}$
}

\author{
Breno C. Barbosa ${ }^{2 *}$, Patrícia M.C. Freitas ${ }^{2}$, Fernanda S. Alves ${ }^{2}$, Lauranne A. Salvato ${ }^{2}$, \\ Marcelo R. Luz ${ }^{2}$, Paulo Ricardo O. Paes ${ }^{2}$, Suzane L. Beier ${ }^{2}$ e Rafael R. Faleiros ${ }^{2}$
}

\begin{abstract}
Barbosa B.C., Freitas P.M.C., Alves F.S., Salvato L.A., Luz M.R., Paes P.R.O., Beier S.L. \& Faleiros R.R. 2017. [Serial administration of 7.5\% hypertonic saline solution in the therapy of severe sepsis due to acute hemorrhagic diarrhea syndrome in dogs.] Administração seriada de salina hipertônica 7,5\% na terapia para sepse grave decorrente da síndrome da diarreia hemorrágica aguda em cães. Pesquisa Veterinária Brasileira 37(9):963-970. Departamento de Clínica e Cirurgia Veterinárias, Escola de Veterinária, Universidade Federal de Minas Gerais, Av. Antonio Carlos 6627, Cx. Postal 567, Campus Pampulha, Belo Horizonte, MG 31270-901, Brazil. E-mail: brenocurty@hotmail.com

The association between acute hemorrhagic diarrhea syndrome and sepsis is frequent in dogs and causes high mortality. In this context we investigated in a randomized single-center controlled trial the late effects of 7.5\% hypertonic saline solution in serial applications on hemodynamic, clinical and laboratory variables in dogs with severe sepsis due to the syndrome. Twelve dogs were randomly distributed into two groups of equal numbers, control (CON) and 7.5\% hypertonic saline solution (SSH). Clinical and laboratory variables were evaluated immediately after admission of patients (T0), 24 (T24), 48 (T48) and 72 (T72) hours after the admission. The SSH group received Ringer with lactate, antibiotic therapy, analgesic and $5 \mathrm{~mL} / \mathrm{kg}^{-1}$ bolus of $7.5 \%$ SSH over 4 minutes on T24 and T48. The CON group received the same therapy, but instead of $7.5 \%$ SSH, Ringer with lactate bolus was given at the same dosis and times. All evaluations were performed prior to the administration of the bolus in both groups. Data collection included complete blood count and clinical variables (AVDN scale, degree of dehydration, respiratory rate and rectal temperature), heart rate (HR) and systolic blood pressure (SBP). Parametric data were evaluated by the Student Newman Keuls and Student $t$ tests, and the nonparametric ones by the Friedman and Mann Whitney test, with a significance level of 0.05 . There were no significant differences between the groups and moments in clinical variables. Nevertheless, the SSH group presented a significant elevation of SBP and HR reduction at T72. Hematocrit and hemoglobin concentration decreased in both groups over time. Total leukocyte and monocyte counts showed a significant elevation in the SSH group, however the leukocytes were within the normal range at T72. There were no significant differences in the segmental neutrophils, but a 9.5-fold increase in T72 compared to T24 $(\mathrm{P}=0.09)$ in the SSH group was observed, whereas this increase was only 2.5 -fold in the $\mathrm{CON}$ group $(\mathrm{P}=0.30)$. A decrease in platelet counts and globulin concentration was observed in the CON group, while these variables remained stable in the SSH group. In conclusion, serial administration of $7.5 \% \mathrm{SSH}$ is promising in the therapy of dogs with acute hemorrhagic diarrhea syndrome, since it assists in the stabilization of leukocytes, platelets and globulins in dogs with severe sepsis due to this syndrome.
\end{abstract}

INDEX TERMS: Acute hemorrhagic diarrhea syndrome, dogs, hypertonic saline, severe sepsis, terapy.

${ }^{1}$ Recebido em 6 de março de 2016.

Aceito para publicação em 30 de dezembro de 2016.

${ }^{2}$ Departamento de Clínica e Cirurgia Veterinárias, Escola de Veterinária,

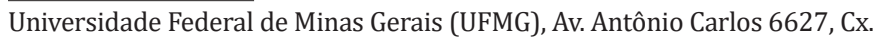
Postal 567, Campus Pampulha, Belo Horizonte, MG 31270-901, Brasil. Pesquisa de mestrado. *Autor para correspondência: brenocurty@hotmail.com 
RESUMO.- Na clínica de animais de companhia é frequente cães com síndrome da diarreia hemorrágica aguda associada a quadros de sepse, o que acarreta alta mortalidade. Nesse contexto, objetivou-se, em um ensaio clínico controlado aleatorizado de centro único, estudar os efeitos tardios da solução salina hipertônica a 7,5\% em aplicações seriadas, sobre variáveis hemodinâmicas, clínicas e laboratoriais em cães com quadro de sepse grave decorrente desta síndrome. Para tal, 12 cães foram aleatoriamente distribuídos em dois grupos de igual número, sendo um controle (CON) e o outro, solução salina hipertônica 7,5\% (SSH). Variáveis clínicas e laboratoriais foram avaliadas imediatamente após a admissão do paciente (T0), 24 (T24), 48 (T48) e 72 (T72) horas após a admissão. 0 grupo SSH recebeu Ringer com lactato, antibioticoterapia, analgésico e SSH 7,5\% em bolus (5mL kg-1 em 4 minutos) no T24 e no T48. 0 grupo CON recebeu a mesma terapia acima, porém ao invés da utilização de SSH a 7,5\%, administrou-se bolus de solução de Ringer lactato na mesma dose e tempos utilizado. As avaliações em cada tempo foram realizadas anteriormente à administração dos bolus, nos dois grupos. Avaliaram-se hemograma completo e as variáveis clínicas (escala AVDN, grau de desidratação, frequência respiratória e temperatura retal), frequência cardíaca (FC), pressão arterial sistólica (PAS). Os dados paramétricos foram avaliados pelos testes Student Newman Keuls e teste t de Student, e os não paramétricos pelo teste de Friedman e Mann Whitney, com nível de significância de $\mathrm{P} \leq 0,05$. Nas variáveis clínicas estudadas não se observou diferença entre os grupos e entre os momentos avaliados. Observou-se diferença significativa no grupo SSH no T72, com elevação da PAS e redução da FC, fato não observado no grupo $\mathrm{CON}$, onde esses parâmetros não se alteraram. 0 hematócrito e a concentração de hemoglobina diminuíram em ambos os grupos com o tempo. As contagens dos leucócitos totais e dos monócitos apresentaram uma elevação significativa no grupo SSH, estando os leucócitos dentro da faixa de normalidade no T72. Não houve diferenças significativas em relação aos neutrófilos segmentados, porém no grupo SSH verificou-se aumento de 9,5 vezes no T72 comparado com o T24 $(\mathrm{P}=0,09)$, enquanto que este aumento foi de apenas 2,5 vezes no grupo CON $(\mathrm{P}=0,30)$. Observou-se ainda redução nas contagens de plaquetas e na concentração de globulinas no grupo COM, enquanto essas variáveis se mantiveram estáveis no grupo SSH. Conclui-se que a administração seriada de SSH $7,5 \%$ se mostrou promissora no tratamento de cães com síndrome da diarreia hemorrágica aguda, pois auxilia na estabilização dos leucócitos, plaquetas e globulinas de cães com sepse grave decorrente da síndrome da diarreia hemorrágica aguda.

TERMOS DE INDEXAÇÃO: Diarreia hemorrágica aguda, cães, salina hipertônica, sepse, terapia.

\section{INTRODUÇÃO}

Na clínica de animais de companhia é alta a frequência de cães com síndrome da diarreia hemorrágica aguda, e geralmente essa cursa com uma elevada taxa de mortalidade, por evoluir para quadros de sepse (Unterer et al., 2014).
De acordo com Dellinger et al. (2013), a sepse é definida como uma resposta sistêmica e deletéria do hospedeiro a uma infecção, e pode ser classificada em quadros conforme os sinais clínicos que o paciente apresenta. Sendo assim, estratificada em síndrome da resposta inflamatória sistêmica (SIRS), síndrome sepse, sepse grave e choque séptico. Sendo que a evolução ocorre da SIRS para choque séptico, respectivamente, e essa evolução, segundo Silverstein $\&$ Sanotoro-Beer (2013), se deve a intensidade em que se desenvolve o desequilíbrio no organismo animal entre a produção e liberação das citocinas pró e anti-inflamatórias. A sepse grave é definida como sepse associada a uma disfunção orgânica ou a hipoperfusão tecidual induzida pela sepse (Dellinger et al., 2013).

Nos quadros de sepse a restituição volêmica deve ser realizada imediatamente para aumentar o sucesso no tratamento, pois proporciona uma melhora na perfusão tecidual, garantindo maior oferta de oxigênio e nutrientes aos tecidos, com menor taxa de apoptose celular e liberação de radicais livres. Essa reposição volêmica, quando realizada de forma controlada, permite restituição do quadro hemodinâmico e pressórico sem produzir maiores riscos de formação de edemas sistêmicos (Durairaj \& Schmidt, 2008).

Dentre as substâncias capazes de repor volemia em tempo curto e com menor volume se destaca a solução salina hipertônica (SSH) com seu efeito hemodinâmico bastante esclarecido na literatura (Velasco et al. 1980, Friedman et al. 2008, Strano et al. 2010). Entretanto, sabe-se que a SSH pode apresentar, além das características expansoras vasculares, ação anti-inflamatória, redução do edema das células endoteliais, permitindo assim um maior fluxo sanguíneo aos tecidos (Jungeret al. 1994, Coimbra et al. 1995). Além disso, possibilita uma melhora na função cardíaca e controle dos quadros de acidose metabólica (Velasco et al. 1980, Strano et al. 2010).

Frente aos possíveis efeitos sistêmicos da SSH, objetivou-se com estudar os efeitos tardios da solução salina hipertônica a 7,5\% em aplicações seriadas, sobre variáveis hemodinâmicas, clínicas e laboratoriais em cães com quadro de sepse grave decorrente da síndrome da diarreia hemorrágica aguda.

\section{MATERIAL E MÉTODOS}

Utilizou-se de um ensaio clínico controlado aleatorizado de centro único (Hospital Veterinário da UFMG) aprovado pelo Comitê de Ética no Uso de Animais (CEUA), da Universidade Federal de Minas Gerais, sob o protocolo nํㅜ 259/2015.

Animas. Foram selecionados 12 animais dentre os cães atendidos na rotina clínica do Setor de Emergência e Terapia Intensiva no Hospital Veterinário da Universidade Federal de Minas Gerais (HV-UFMG) com síndrome da diarreia hemorrágica aguda. Os animais foram distribuídos em dois grupos, de igual número $(n=6)$, de forma aleatória via sorteio prévio, sendo grupo controle (CON) e grupo solução salina hipertônica (SSH). O grupo CON constou das seguintes médias e desvios padrão para as variáveis peso e idade, respectivamente: 5,78 $\pm 4,33 \mathrm{em} \mathrm{kg}$ e 3,6 $\pm 1,5$ em meses, e o grupo SSH 5,99 $\pm 3,87 \mathrm{~kg}$ e 4,16 $\pm 2,4$ meses, sem diferença entre os grupos. Os animais inclusos possuíam resultado positivo para o teste rápido de antígeno canino para parvovirose/coronavi$\operatorname{rose}^{1}$ (AleneBioEasy ${ }^{\circledR}$ ) via swab retal, até 12 meses de idade, no 
máximo 48 horas do início dos sintomas e presença de ao menos dois dos critérios descritos para SIRS em cães (Quadro 1) e pelo menos uma disfunção orgânica (Quadro 2), diagnosticados assim em sepse grave. Também foram realizadas a determinação do lactato sérico (dosado pelo aparelho analisador químico da Roche Diagnostic Systems ${ }^{\circledR}$, por meio do método colorimétrico) nos animais de ambos os grupos, no qual foi encontrado no grupo CON $2,71 \pm 1,04 \mathrm{mmol} / \mathrm{L}$, e no grupo SSH 2,99 $\pm 1,0 \mathrm{mmol} / \mathrm{L}$, sem diferença significativa entre ambos (teste t de Student, $\mathrm{P} \leq 0,05$ ).

Tratamento. Assim que admitidos, todos os cães, independentemente do grupo, foram submetidos ao protocolo padrão de tratamento realizado no Setor de Emergência e Terapia Intensiva da EV-UFMG. Este protocolo consiste de fluidoterapia para adequação do equilíbrio hidroeletrolítico, ácido-base e energético, antibioticoterapia, terapia antiálgica e antiemética. A fluidoterapia foi estabelecida com solução de Ringer com lactato via bomba de infusão volumétrica (FreseniusKabi ${ }^{\circledR}$ ) por cateter venoso central (Duocath ${ }^{\circledR}$ ) com gauge variável ao porte do paciente. 0 volume e taxa de infusão foram baseados no grau de desidratação do animal em que se calculou o requerimento de fluidoterapia multiplicando o peso pela porcentagem de desidratação, o resultado final se deu em litros (L), sendo administrado entre 12-24 horas. Consideraram-se parâmetros clínicos para cálculo da porcentagem de desidratação: paciente hidratado, menor que $5 \%$, cursando sem alterações clínicas; desidratação leve, próxima de 5\%, sinais de mínima perda do turgor cutâneo, pouco ressecamento de mucosas, normalidade do globo ocular; desidratação moderada, próxima de 8\%, moderada perda do turgor cutâneo, mucosas ressecadas, pulso periférico acelerado e enolftalmia; desidratação grave, maior que $10 \%$, perda do turgor cutâneo, enolftalmia grave, taquicardia, mucosas extremamente ressecadas, pulso fraco e filiforme, hipotensão e alteração no nível de consciência. Visando rápida restituição volêmica, um quarto do volume total diário foi reposto nas primeiras quatro horas. Nos animais que apresentavam hipotensão, com valor de PAM inferior a $65 \mathrm{mmHg}$ ou PAS

\section{Quadro 1. Valores de variáveis fisiológicas em cão com quadro de síndrome da resposta inflamatória sistêmica (SIRS)}

\begin{tabular}{lc}
\hline Variável & Cão \\
\hline Temperatura retal & $<38,1^{\circ} \mathrm{C}$ ou $>39,2^{\circ} \mathrm{C}$ \\
Frequência cardíaca & $>120 \mathrm{bpm}$ \\
Frequência respiratória & $>20 \mathrm{mpm}$ \\
Leucócitos totais; bastonetes & $<6 \times 103 / \mathrm{mm} 3$ ou $>16 \times 103 / \mathrm{mm} 3 ;>3 \%$
\end{tabular}

Fonte: Hauptmann et al. (1997).

\section{Quadro 2. Disfunções orgânicas associadas ao quadro de sepse grave em cão}

Hipotensão: PAM < 80mmHg ou PAS < $120 \mathrm{mmHg}$

Hipotensão grave: queda abrupta de mais de $40 \mathrm{mmHg} g$

na PAS ou PAM $<65 \mathrm{mmH}$

Oligúria $(<2 \mathrm{~mL} / \mathrm{kg} / \mathrm{h})$ ou Creatinina $>2 \mathrm{mg} / \mathrm{dL}$

Hiperbilirrubinemia: $>0,5 \mathrm{mg} / \mathrm{dL}$

Consciência alterada: Glasgow pediátrico modificado $<17$ ou AVDN $<$ A Disfunção respiratória

Trombocitopenia: $<50.000 / \mathrm{mm} 3$ ou queda de mais de $50 \%$ em 12 horas

Aumento de TP, TTPA/D-dímero ou queda de fibrinogênio Íleo paralítico

Albumina: $<2,5 \mathrm{~g} / \mathrm{dL}$

PAM: pressão arterial média; PAS: pressão arterial sistólica; AVDN: alerta, responsivo a comando verbal, responsivo à dor, não responsivo; TP: tempo de protrombina; TTPA: tempo de tromboplastina ativada. Adaptado de Rabelo (2013). inferior a $90 \mathrm{mmHg}$, utilizou-se a técnica de desafios volêmicos como descrito por Mensack (2008) e por Davis et al. (2013). Para tanto, era administrado $10 \mathrm{~mL} \mathrm{~kg}^{-1}$ de solução Ringer com lactato durante seis minutos seguidos por avaliação constante da PAS. Caso o quadro pressórico não fosse reestabelecido, um novo desafio volêmico era realizado e mais uma vez monitorado continuamente. Se a PAS não se apresentasse dentro da normalidade após o terceiro desafio, agentes vasopressores e inotrópicos eram administrados e o cão era excluído do experimento.

Além do tratamento descrito acima, os animais do grupo SSH receberam duas aplicações de solução de salina hipertônica a 7,5\% (5mL.kg-1, no tempo de quatro minutos, IV, em bomba de infusão). As aplicações foram realizadas 24 e 48 horas depois do início do tratamento, logo após as colheitas de amostras sanguíneas descritas adiante.

Procedimentos. Imediatamente após o sorteio para determinação do grupo (T0), 24 (T24), 48 (T48) e 72 (T72) horas após a instituição da terapia de suporte, todos os animais foram submetidos à inspeção, para determinar o nível de consciência via escala AVDN, e ao exame físico. No sistema AVDN, qualquer classificação diferente de A (Alerta) foi considerada como alteração neurológica e, portanto, uma disfunção orgânica. Para permitir a comparação entre grupos por este método, foram atribuídos escores para cada item da escala, sendo que o A (alerta) recebeu o número 1; $\mathrm{V}$ (responsivo a voz) o número 2; $\mathrm{D}$ (responsivo a dor) o número 3; N (não responsivo) o número 4. Para o exame físico foram mensurados a frequência cardíaca (FC) via auscultação e eletrocardiograma digital (eletrocardiógrafo digital ECG PC veterinário, $\mathrm{TEB}^{\circledR}$ ), a frequência respiratória (f) via auscultação, a temperatura retal (TR) por termômetro digital e a pressão arterial sistólica por doppler vascular (Doppler Vascular Veterinário 812, Park Medical Eletronics ${ }^{\circledR}$ ).

Além dessas variáveis foram analisadas a coloração de mucosas orais e oculares via inspeção, o turgor cutâneo, o tempo de preenchimento capilar (TPC) da mucosa oral, a qualidade de pulso por palpação digital das artérias femorais, a saturação de oxihemoglobina por oxímetro portátil $\left(\mathrm{OxiTouch}^{\circledR}\right)$ posicionado na pina auricular ou dígito do paciente e a presença de dor e tensão via palpação abdominal.

Na admissão (T0), obteve-se uma amostra de sangue arterial coletada da artéria metatarsiana para análise da oxigenação tecidual por meio do cálculo da relação da pressão parcial de oxigênio arterial com a fração inspirada de oxigênio $\left(\mathrm{PaO}_{2} \mathrm{FiO}_{2}{ }^{-1}\right)$.

Nos mesmos tempos correspondentes ao exame clínico (T0 a T72), realizou-se a coleta de 1,5 mL de sangue através das veias cefálicas ou jugulares externas para a realização dos exames laboratoriais. Após a coleta do sangue este foi fracionado e acondicionado em tubos com anticoagulante EDTA, e em tubos sem anticoagulante para a realização de exames laboratoriais.

Em todas as amostras sanguíneas, realizou-se hemograma completo por meio do aparelho Abacus Junior Vet $\left(\right.$ ARCUS $^{\circledR}$ ), onde se determinaram a contagem de hemácias, volume corpuscular médio (VCM), concentração de hemoglobina corpuscular média (CHCM), hemoglobina corpuscular média, leucograma, plaquetas, albumina e globulinas.

Análise estatística. Os dados foram agrupados e submetidos a ANOVA, para verificação de distribuição de normalidade. Os dados paramétricos foram submetidos a análise de variância em blocos ao acaso, seguido pelo teste de Student Newman Keuls, para verificar o efeito do tempo em cada grupo e pelo teste $t$ de Student para verificar o efeito do tratamento em cada tempo. As variáveis que não apresentaram distribuição normal (contagem de monócitos, frequência respiratória e concentração plasmática de proteínas totais) passaram por transformação logarítmica prévia. Os dados não paramétricos foram analisados pelo teste de 
Friedman para verificar o efeito do tempo em cada grupo e pelo teste de Mann Whitney para verificar o efeito do tratamento em cada tempo. Para todas as análises considerou-se o nível de significância $\mathrm{P} \leq 0,05$.

\section{RESULTADOS E DISCUSSÃO}

A solução de salina hipertônica tem sido amplamente utilizada na emergência veterinária devido a seus efeitos hemodinâmicos positivos em animais que necessitam de reposição volêmica imediata. Entretanto, raros ainda são os estudos sobre seus efeitos tardios sobre hemograma completo, variáveis clínicas e hemodinâmicas. Após ampla revisão da literatura nacional e internacional, não foram encontrados estudos sobre o uso clínico dessa solução com o intuito de estimular a produção celular medular em pacientes leucopênicos, com o uso da salina hipertônica de forma sequencial e tardia em cães. Os animais do presente experimento foram selecionados entre 116 cães atendidos com síndrome da diarreia hemorrágica aguda no HV-UFMG, no período de junho de 2014 a julho de 2015. Todos os 12 animais $(10,3 \%)$ selecionados atenderam completamente os critérios de inclusão. As causas de exclusão dos demais foram idade superior a 12 meses $(18,96 \%)$ ou presença de sinais clínicos há mais de 3 dias $(9,48 \%)$, ausência de disfunção orgânica $(16,3 \%)$ ou choque séptico $(6,89 \%)$, impossibilidade de internação $(18,10 \%)$, presença de corpo estranho intestinal (6,89\%), diagnóstico de giardíase ou outras afecções $(7,75 \%)$, e antibioticoterapia prévia $(5,17 \%)$.

Ressalta-se que os pacientes foram monitorados continuamente e receberam o tratamento suporte até a sua alta hospitalar, onde não se constatou nenhum caso de óbito. As coletas de dados e dos exames do presente estudo foram realizadas de forma pontuais, a fim de padronização do experimento. Tal metodologia não reflete a realidade atual do setor, pois a fluidoterapia e o suporte médico veterinário são feitos de forma constante durante 24 horas por dia.

$\mathrm{PaO}_{2} / \mathrm{FiO}_{2}$. Os resultados iniciais da oxigenação tecidual por meio da avaliação gasométrica do sangue arterial foram $324,34 \pm 64,7$ e $348,41 \pm 37,23$ para os grupos CON e SSH, respectivamente. Não houve diferença significativa entre os grupos $(\mathrm{P}>0,05)$, fato que demonstra a homogeneidade entre os grupos no momento do início do tratamento. A avaliação inicial da oxigenação tecidual realizada neste estudo foi importante, pois sabe-se que o pulmão é um órgão que frequentemente evolui com disfunção nos animais com sepse, mesmo que o foco infeccioso não seja pulmonar. Isso ocorre provavelmente pela presença de mediadores inflamatórios na circulação sistêmica. Durante a sepse, a barreira alveolocapilar pode estar lesada, permitindo que citocinas e outras proteínas cheguem ao alvéolo. Isso resulta em aumento de permeabilidade, causando aumento do influxo de proteínas plasmáticas e células, que levam à inativação do surfactante pulmonar (Ware \& Matthay, 2000). Com isso, os animais desenvolvem quadro da Síndrome da Angústia Respiratória Aguda (SARA) leve, moderada ou grave, que acarretam disfunção pulmonar grave devido a quadros de hipóxia tecidual. 0 grau de hipóxia tecidual pode ser verificado pela hemogasometria arterial, obtendo a relação $\mathrm{PaO}_{2} \mathrm{FiO}_{2}^{-1}$. Na SARA leve, essa relação assume valores maiores que $200 \mathrm{mmHg}$ e menores ou igual a $300 \mathrm{mmHg}$. Já na SARA moderada é inferior ou igual a $200 \mathrm{mmHg}$, e maior que $100 \mathrm{mmHg}$, e na grave menor ou igual a $100 \mathrm{mmHg}$ (Serio \& Consorti 2013, Bernard \& Artigas 2016, Sorbo et al. 2016). Assim, como observado nos resultados descritos acima, nesse estudo os animais não apresentavam SARA.

AVDN. Empregada com objetivo de verificar a homogeneidade dos grupos. No Quadro 3 observa-se os valores da variável em cada tempo por grupo. Não houve diferença significativa entre os grupos.

Variáveis clínicas. As variáveis clínicas estão demonstradas no Quadro 4. A variável desidratação também foi utilizada para demonstrar homogeneidade entre os grupos. Quanto maior o valor da média, maior o quadro de desidratação. Observa-se uma semelhança entre os dois grupos, pois não houve diferença significativa entre eles, porém em ambos verifica-se uma redução significativa entre os tempos a partir de 24 horas, oriunda da expansão volêmica após a admissão. Em ambos os grupos, observou-se melhora clínica dos animais no decorrer das avaliações. Entretanto, somente no grupo SSH observou-se redução significativa na frequência cardíaca e elevação na PAS (Quadro 4). A elevação da PAS no grupo SSH propiciou a redução na FC, sendo este efeito benéfico. Esse achado provavelmente foi decorrente do uso da SSH, que segundo Engen (2006), favorece o débito cardíaco.

Assim, acredita-se que a administração de SSH 7,5\% apresentou efeito positivo sobre a PAS, que se refletiu em redução da FC. Segundo Engen (2006), a pressão arterial é dependente e controlada pela frequência cardíaca (coração) e o sistema endócrino. Logo, quanto maior a pressão arterial, menor será a frequência cardíaca para manter o débito cardíaco adequado.

Não foram observadas diferenças significativas entre os grupos para frequência respiratória. As alterações respiratórias em sepse geralmente estão associadas com quadros de SARA. No momento da admissão foi realizada a relação $\mathrm{PaO}_{2} \mathrm{FiO}_{2}^{-1}$, estando dentro dos valores de normalidade.

Também não se observaram diferenças significativas dentro dos grupos, para temperatura retal, que se manteve dentro da normalidade. Além disso, não houve diferença na taxa de mortalidade (igual a zero) e na sobrevida dos pacientes entre os grupos. Haja vista que após 60 dias da alta hospitalar, todos os animais se apresentavam clinicamente recuperados.

Hematimetria. Os valores da hematimetria estão representados no Quadro 5. No momento da admissão, os

\begin{tabular}{|c|c|c|c|c|}
\hline $\begin{array}{l}\text { Nível consciência } \\
\text { (grau) }\end{array}$ & T0 & $\mathrm{T} 24$ & $\mathrm{~T} 48$ & $\mathrm{~T} 72$ \\
\hline Grupo CON & $2,17^{\mathrm{Aa}} \pm 0,69$ & $1,67^{\mathrm{Aa}} \pm 0,47$ & $1,33^{\mathrm{Aa}} \pm 0,47$ & $1,0^{\mathrm{Aa}} \pm 0,0$ \\
\hline Grupo SSH & $2,17^{\text {Aa }} \pm 0,69$ & $1,17^{\mathrm{Aa}} \pm 0,37$ & $1,17^{\mathrm{Aa}} \pm 0,37$ & $1,0^{\mathrm{Aa}} \pm 0,0$ \\
\hline
\end{tabular}

Médias seguidas por letras maiúsculas e minúsculas iguais não diferem entre si entre colunas e linhas respectivamente $(\mathrm{P} \leq 0,05)$. 


\begin{tabular}{|c|c|c|c|c|c|}
\hline Variável & & T0 & $\mathrm{T} 24$ & $\mathrm{~T} 48$ & $\mathrm{~T} 72$ \\
\hline \multirow[t]{2}{*}{ Desidratação (\%) } & Controle & $9 \pm 1^{\mathrm{Aa}}$ & $7 \pm 2^{\mathrm{Ab}}$ & $6 \pm 1^{\mathrm{Ab}}$ & $5 \pm 0^{\mathrm{Ab}}$ \\
\hline & SSH & $9 \pm 1^{\mathrm{Aa}}$ & $6 \pm 1^{\mathrm{Ab}}$ & $6 \pm 1^{\mathrm{Ab}}$ & $5 \pm 0^{\mathrm{Ab}}$ \\
\hline \multirow[t]{2}{*}{ FC (bpm) } & Controle & $167,3 \pm 21,2^{\mathrm{a}}$ & $165,8 \pm 39,7^{\mathrm{a}}$ & $163,2 \pm 33,3^{\mathrm{a}}$ & $139,2 \pm 15,8^{a}$ \\
\hline & SSH & $175,0 \pm 24,0^{\mathrm{a}}$ & $133,8 \pm 29,0^{\mathrm{b}}$ & $129,5 \pm 29,5^{b}$ & $113,0 \pm 13,9^{b}$ \\
\hline \multirow[t]{2}{*}{ PAS (mmHg) } & Controle & $86 \pm 28^{a}$ & $111 \pm 24 a$ & $97,7 \pm 21,2^{\mathrm{a}}$ & $105 \pm 10,7^{a}$ \\
\hline & SSH & $85,3 \pm 21,4^{\mathrm{b}}$ & $100,2 \pm 7,5^{\mathrm{a}}$ & $104,2 \pm 10,4^{\mathrm{a}}$ & $109,3 \pm 7,5^{\mathrm{a}}$ \\
\hline \multirow[t]{2}{*}{$\mathrm{f}(\mathrm{mpm})$} & Controle & $33,0 \pm 11,4$ & $30,3 \pm 14,0$ & $25,3 \pm 8,7$ & $23,3 \pm 8,0$ \\
\hline & SSH & $42,7 \pm 23,4$ & $27,0 \pm 10,0$ & $26,2 \pm 4,5$ & $29,0 \pm 15,0$ \\
\hline \multirow[t]{2}{*}{$\mathrm{TR}\left(\mathrm{C}^{\circ}\right)$} & Controle & $38,6 \pm 1,2$ & $38,8 \pm 0,4$ & $38,2 \pm 0,3$ & $38,2 \pm 0,6$ \\
\hline & SSH & $38,7 \pm 1,5$ & $38,2 \pm 0,4$ & $38,3 \pm 0,6$ & $38,3 \pm 0,2$ \\
\hline
\end{tabular}

Médias seguidas por letras maiúsculas e minúsculas iguais não diferem entre si entre colunas e linhas respectivamente $(\mathrm{P} \leq 0,05)$.

Quadro 5. Médias e desvios-padrão das variáveis de hematimetria de cães submetidos a terapia para síndrome da diarreia hemorrágica aguda associada (SSH) ou não (CON) à aplicação seriada de solução salina hipertônica

\begin{tabular}{lccccc}
\hline Variável & & $\mathrm{T} 0$ & $\mathrm{~T} 24$ & $\mathrm{~T} 48$ & $\mathrm{~T} 72$ \\
\hline Hematócrito (\%) & Controle & $41,7 \pm 5,1^{\mathrm{Aa}}$ & $31,8 \pm 3,1^{\mathrm{Ab}}$ & $32,0 \pm 4,9^{\mathrm{Ab}}$ & $29,5 \pm 3,9^{\mathrm{Ab}}$ \\
& SSH & $34,7 \pm 7,2^{\mathrm{Aa}}$ & $32,2 \pm 7,5^{\mathrm{Ab}}$ & $28,8 \pm 4,7^{\mathrm{Ab}}$ & $28,2 \pm 5,4^{\mathrm{Ab}}$ \\
Hemoglobina & Controle & $13,8 \pm 2,1^{\mathrm{Aa}}$ & $10,2 \pm 1,4^{\mathrm{Ab}}$ & $10,1 \pm 1,^{\mathrm{Ab}}$ & $9,6 \pm 1,8^{\mathrm{Ab}}$ \\
& SSH & $11,5 \pm 2$, $^{\mathrm{Aa}}$ & $10,3 \pm 2,8^{\mathrm{Aa}}$ & $9,5 \pm 1,6^{\mathrm{Ab}}$ & $9,0 \pm 2,0^{\mathrm{Ab}}$ \\
Hemácias & Controle & $6,0 \pm 1,8$ & $5,0 \pm 0,5$ & $4,9 \pm 0,9$ & $4,0 \pm 0,6$ \\
$\left(1 \times 10^{6} \mathrm{cel} / \mathrm{ul}\right)$ & SSH & $5,3 \pm 1,0$ & $5,0 \pm 1,0$ & $4,4 \pm 0,6$ & $4,3 \pm 0,8$ \\
VCM (fl) & Controle & $81,9 \pm 4,3$ & $62,8 \pm 4,8$ & $62,6 \pm 3,5$ & $61,8 \pm 2,6$ \\
& SSH & $65,4 \pm 2,4$ & $64,3 \pm 2,3$ & $64,9 \pm 2,5$ & $64,1 \pm 3,1$ \\
CHCM (g/dL) & Controle & $32,8 \pm 1,3$ & $32,4 \pm 3,0$ & $32,5 \pm 2,5$ & $32,9 \pm 2,6$ \\
& SSH & $33,1 \pm 0,8$ & $31,9 \pm 1,3$ & $33,0 \pm 0,3$ & $32,9 \pm 2,2$ \\
HCM (pg) & Controle & $27,0 \pm 1,4$ & $20,3 \pm 1,4$ & $20,0 \pm 1,3$ & $20,4 \pm 1,3$ \\
& SSH & $21,7 \pm 1,2$ & $20,7 \pm 1,4$ & $22,1 \pm 1,6$ & $21,1 \pm 1,1$
\end{tabular}

Médias seguidas por letras maiúsculas e minúsculas iguais não diferem entre si entre colunas e linhas respectivamente $(\mathrm{P} \leq 0,05)$.

animais se encontravam em desidratação, justificando os maiores valores de hematócrito e concentração de hemoglobina em T0. Não se verificaram diferenças entres grupos em qualquer tempo, contudo, houve redução nas médias de ambos os grupos durante o período experimental já a partir de T24. Tais resultados são reflexos da terapia de restituição volêmica, que foi adequada em ambos os grupos.

Os valores de normalidade para hematimetria são: he-

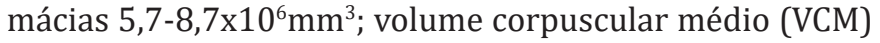
60-77fl; concentração de hemoglobina corpuscular média (CHCM) 32-36\%; hemoglobina corpuscular média (HCM) 19-23pg (Garcia-Navarro 2005). Não se observaram quaisquer diferenças significativas relativas a essas variáveis. Entretanto, as médias de contagens de hemácias permaneceram abaixo da faixa de normalidade a partir de T24 em ambos os grupos. Acredita-se que este fato se deva ao parvovírus canino, que apresenta tropismo pelas células das linhagens progenitoras eritróides (Mendes et al. 2011). Os valores normais verificados em T0 se justificam pelo quadro de depleção volêmica gerando uma hemoconcentração. Segundo Mendes et al. (2011), que avaliaram perfil hematológico e bioquímico de cães com gastroenterite hemor- rágica, não foram observadas diferenças significativas nos valores de VCM, CHC e HCM, como também observado no presente estudo, permanecendo dentro dos valores de normalidade.

Leucograma. Os dados do leucograma estão representados no quadro 6. Ambos os grupos apresentam contagens de leucócitos abaixo do normal para a espécie na admissão (T0), já que a faixa de normalidade é de 6.000 a $17.000 \mathrm{~mm}^{3}$ (Jain 1993). Essas baixas contagens de leucócitos devem ter sido decorrentes da síndrome da diarreia hemorrágica aguda, já que nessa enfermidade observa-se um intenso tropismo viral para células em multiplicação do organismo, com destaque para os leucócitos (Mendes et al. 2011). Neste estudo também se pôde verificar leucopenia até dois dias após o início do tratamento em 100\% dos animais, o que coincide com o relatado por Kogika et al. (2003). Tais autores descreveram que a leucopenia está presente em $60 \%$ dos casos na avaliação sanguínea inicial, passando para $100 \%$ no segundo e terceiro dias de cães com síndrome da diarreia hemorrágica aguda. Entretanto, pode-se observar que nos animais do grupo SSH houve um aumento gradual nos valores dessa variável no decorrer dos momentos, encontrando-se no T72 valores dentro 
Quadro 6. Médias e desvios-padrão das variáveis de leucograma de cães submetidos a terapia para síndrome da diarreia hemorrágica aguda associada (SSH) ou não (CON) à aplicação seriada de solução salina hipertônica

\begin{tabular}{|c|c|c|c|c|c|}
\hline Variável & & T0 & T24 & T48 & $\mathrm{T} 72$ \\
\hline Leucócitos totais & Controle & $2686 \pm 2969^{a}$ & $1301 \pm 871^{\mathrm{a}}$ & $2097 \pm 1278^{a}$ & $4345 \pm 2471^{a}$ \\
\hline$\left(\right.$ cels $/ \mathrm{mm}^{3}$ ) & $\mathrm{SSH}$ & $3152 \pm 2591^{\mathrm{ab}}$ & $2518 \pm 973^{b}$ & $3853 \pm 2894^{\mathrm{ab}}$ & $6755 \pm 4304^{a}$ \\
\hline Segmentados & Controle & $1316 \pm 1937$ & $971 \pm 1746$ & $1074 \pm 758$ & $2471 \pm 2226$ \\
\hline$\left(\right.$ cels $/ \mathrm{mm}^{3}$ ) & $\mathrm{SSH}$ & $1948 \pm 2086$ & $352 \pm 219$ & $1301 \pm 1559$ & $3143 \pm 2949$ \\
\hline Bastonetes & Controle & $151 \pm 260$ & $125 \pm 223$ & $104 \pm 117$ & $98 \pm 91$ \\
\hline (cels $/ \mathrm{mm}^{3}$ ) & $\mathrm{SSH}$ & $0 \pm 0$ & $26 \pm 40$ & $6 \pm 14$ & $35,3 \pm 50,4$ \\
\hline Monócitos & Controle & $214 \pm 213^{a}$ & $190 \pm 195^{a}$ & $284 \pm 413^{a}$ & $257 \pm 190^{a}$ \\
\hline (cels $/ \mathrm{mm}^{3}$ ) & SSH & $535 \pm 345^{\text {ab }}$ & $329 \pm 227^{b}$ & $861 \pm 553^{\text {ab }}$ & $1389 \pm 1127^{a}$ \\
\hline Eosinófilos & Controle & $40 \pm 70$ & $44 \pm 58$ & $12 \pm 23$ & $0 \pm 0$ \\
\hline$\left(\right.$ cels $\left./ \mathrm{mm}^{3}\right)$ & SSH & $136 \pm 254$ & $288 \pm 438$ & $152 \pm 216$ & $182 \pm 269$ \\
\hline Linfócitos & Controle & $40 \pm 70$ & $44 \pm 58$ & $12 \pm 23$ & $0 \pm 0$ \\
\hline$\left(\right.$ cels $/ \mathrm{mm}^{3}$ ) & SSH & $136 \pm 254$ & $288 \pm 438$ & $152 \pm 216$ & $182 \pm 269$ \\
\hline Plaquetas & Controle & $320000 \pm 119017^{a}$ & $147000 \pm 49686^{\mathrm{b}}$ & $190167 \pm 84464^{\mathrm{ab}}$ & $140000 \pm 94251^{b}$ \\
\hline$\left(\right.$ cels $/ \mathrm{mm}^{3}$ ) & SSH & $288667 \pm 134442 a$ & $256000 \pm 166228^{a}$ & $244167 \pm 136792^{a}$ & $171000 \pm 180809^{a}$ \\
\hline
\end{tabular}

Médias seguidas por letras maiúsculas e minúsculas iguais não diferem entre si entre colunas e linhas respectivamente $(\mathrm{P} \leq 0,05)$.

da faixa de normalidade e estatisticamente superiores a T24. Acredita-se que este efeito foi decorrente da ação da administração da SSH que, de acordo com Friedman et al. (2008), promove modulação da resposta leucocitária.

Os neutrófilos segmentados são células importantes para a defesa do organismo, sendo consideradas células de defesa de primeira linha. Apresentam grânulos com enzimas hidrolíticas e substância antibacterianas fundamentais para controle de infecções (Messick 2012) e o valor de normalidade é de 3000-11500 células / $\mathrm{mm}^{3}$ (Garcia-Navarro, 2005). Apesar de não ter ocorrido diferença significativa entre tempos $(\mathrm{P}=0,09)$, verificou-se que os valores de T72 foram 9 vezes superiores em relação ao T24 no grupo SSH. Este mesmo fato ocorreu no grupo CON, porém em proporções bem menores $(2,5$ vezes, $\mathrm{P}=0,30)$. Ressalta-se que a primeira aplicação de SSH 7,5\% foi realizada após a coleta do hemograma no T24, momento esse em que ambos os grupos apresentavam neutropenia acentuada, e somente no grupo 2 os valores sofreram alterações de tal magnitude.

A neutropenia já era esperada nos animais do presente estudo, sendo derivada de dois aspectos principais, a destruição das células progenitoras na medula óssea marrom e a mobilização delas para o lúmen intestinal. Por serem a linha de frente na defesa, a implementação de uma terapia que favoreça o restabelecimento de contagens é de grande valia, pois sua carência pode agravar o quadro séptico.

Com relação às contagens de monócitos, foi evidente a diferença do grupo SSH para o controle. Enquanto no CON não se observaram diferenças significativas, no grupo SSH houve um aumento significativo no T72, que apresentou valores 4,2 vezes superiores aos observados em T24, momento que antecedeu o início do tratamento com SSH.

Os monócitos são os maiores leucócitos da circulação e apresentam íntima relação com os neutrófilos, pois tem origem na mesma célula precursora. Seu número está relacionado principalmente a um estímulo inflamatório, sendo fundamental nesses casos, pois assim que deixam o vaso sanguíneo e chegam aos tecidos ocorre a diferenciação em macrófagos. 0 sistema fagocitário mononuclear (SFM) é
Quadro 7. Médias e desvios-padrão das concentrações de proteínas sanguíneas em cães submetidos a terapia para síndrome da diarreia hemorrágica aguda associada (SSH) ou não (CON) à aplicação seriada de solução salina hipertônica

\begin{tabular}{lccccc}
\hline Variável & & T0 & T24 & T48 & T72 \\
\hline PPT (g/dL) & Controle & $5,1 \pm 1,0^{\mathrm{a}}$ & $3,8 \pm 0,8^{\mathrm{b}}$ & $3,6 \pm 1,0^{\mathrm{b}}$ & $3,2 \pm 1,0^{\mathrm{b}}$ \\
& SSH & $4,7 \pm 1,2^{\mathrm{a}}$ & $3,5 \pm 0,4^{\mathrm{b}}$ & $3,3 \pm 0,1^{\mathrm{b}}$ & $3,2 \pm 0,2^{\mathrm{b}}$ \\
Albumina (g/dL) & Controle & $2,0 \pm 0,7^{\mathrm{a}}$ & $1,3 \pm 0,5^{\mathrm{b}}$ & $1,2 \pm 0,4^{\mathrm{b}}$ & $1,1 \pm 0,4^{\mathrm{b}}$ \\
& SSH & $2,0 \pm 0,4^{\mathrm{a}}$ & $1,4 \pm 0,3^{\mathrm{b}}$ & $1,4 \pm 0,4^{\mathrm{b}}$ & $1,3 \pm 0,5^{\mathrm{b}}$ \\
Globulinas (g/dL) & Controle & $3,1 \pm 0,5^{\mathrm{a}}$ & $2,5 \pm 0,6^{\mathrm{b}}$ & $2,4 \pm 0,7^{\mathrm{b}}$ & $2,2 \pm 0,6^{\mathrm{b}}$ \\
& SSH & $2,6 \pm 1,0^{\mathrm{a}}$ & $2,1 \pm 0,3^{\mathrm{a}}$ & $1,9 \pm 0,5^{\mathrm{a}}$ & $1,9 \pm 0,4^{\mathrm{a}}$
\end{tabular}

Médias seguidas por letras maiúsculas e minúsculas iguais não diferem entre si entre colunas e linhas respectivamente $(\mathrm{P} \leq 0,05)$.

responsável por fagocitose e digestão dos debris celulares, além de apresentar os antígenos aos linfócitos, atuando na resposta imune (Messick, 2012). Seus valores de normalidade são de 150-1350 células / mm³ (Garcia-Navarro, 2005).

Os macrófagos, monócitos imaturos na corrente sanguínea, apresentam grandes funções como secretar interleucinas, promover resposta inata e adaptativa, controle inflamatório e reparo tecidual, funções primordiais no paciente séptico, além de promover a mobilização dos neutrófilos para a circulação. Essas células possuem capacidade de se transformarem de M1 (células que produzem citocinas pró-inflamatórias) em células M2 (anti-inflamatórias) modulando a inflamação (Tizard, 2013). Afirma-se que a SSH $7,5 \%$ apresenta um efeito positivo, ou seja, estimula a elevação dos monócitos nos cães com síndrome da diarreia hemorrágica aguda após sua aplicação.

Não foram encontradas diferenças significativas nos valores dos eosinófilos entre os grupos, nem dentro de cada grupo com o decorrer do tempo. A normalidade destas células é de 100-1250 células $/ \mathrm{mm}^{3}$ (Garcia-Navarro, 2005). Eosinopenia é comum nos casos de gastroenterite hemorrágica oriunda da liberação endógena dos corticosteróides (Mendes et al. 2011), justificando assim os quadros de eosinopenia do grupo CON e os valores sempre dentro da normalidade no grupo $\mathrm{SSH}$. 
Os valores de referência para contagens de linfócitos são de 1000 a 4800 células $/ \mathrm{mm}^{3}$ (Garcia-Navarro 2005). Não houve diferenças significativas, apenas uma linfopenia em ambos os grupos. Quadros de linfopenia são mais comum nas infecções por coronavírus do que por parvovírus, porém é rotineira a associação de ambos os agentes na síndrome (Ferreira et al. 2004).

Quanto aos valores das plaquetas, observou-se redução gradual dos valores durante os momentos, contudo diferença significativa só esteve presente no grupo CON. Este achado corrobora para uma ação positiva da terapia com SSH 7,5\%. Em geral, a trombocitopenia é um achado variável nos pacientes com a síndrome da diarreia hemorrágica aguda, pois mesmo ocorrendo uma interação do agente com o megacariócito, os valores podem estar próximos da normalidade (200.000-900.000 células $\left./ \mathrm{mm}^{3}\right)$, como descreveram Mendes et al. (2011).

Proteínas plasmáticas. As proteínas plasmáticas (Quadro 7) incluem a albumina e as globulinas, através delas se obtém a relação entre o hematócrito e o estado de hidratação do paciente, sendo que em casos de desidratação os valores de proteína total e hematócrito estão aumentados. Em análise do perfil das proteínas plasmáticas, observou-se redução em ambos os grupos das PTT e da albumina após o T24, fato derivado da restituição volêmica que esses pacientes passaram após o período de admissão. Reforça-se a homogeneidade dos grupos, haja vista que não houve diferença entre os grupos, e em ambos a redução desses valores foram significativas e no mesmo tempo. Os valores de normalidade são $5,7-7,1 \mathrm{~g} \mathrm{dL}^{-1}$ para PPT, 2,6-3,3g dL $\mathrm{g}^{-1}$ para albumina e $2,7-4,4 \mathrm{~g} \mathrm{dL}^{-1}$ para globulinas (Kaneko 1989).

Em relação as globulinas, houve redução numérica em ambos os grupos, entretanto de forma significativa apenas no grupo CON. Essa redução era esperada devido à restituição volêmica, na qual se associa provas de cargas para restaurar o volume plasmático (Davis et al. 2013). Animais que cursam a síndrome comumente apresentam baixos valores de proteínas totais e globulinas por perdas intestinais associado com a inapetência, e uma queda menos acentuada dos valores de albumina sérica por mecanismo de compensação hepática visando manter a osmolaridade plasmática. A perda da integridade da barreira intestinal propicia também a perda da albumina para o lúmen acentuando o quadro hipoprotéico (Mendes et al. 2011).

Entretanto, a ausência de diferença significativa no grupo SSH indica efeito benéfico da administração de SSH 7,5\% sobre o sistema imunológico. As globulinas possuem duas funções básicas no organismo, a primeira de se ligar as moléculas do agente invasor para induzir resposta imunológica, e a segunda de funcionar como recruta de outras células para destruição do patógeno, sendo um importante mecanismo de defesa (Janeway 2007).

\section{CONCLUSÃO}

A administração seriada de SSH 7,5\%, 24 e 48 horas após o início da terapia padrão, se mostrou promissora no tratamento de cães com síndrome da diarreia hemorrágica aguda, pois auxilia na estabilização dos leucócitos, plaque- tas e globulinas de cães com sepse grave decorrente desta síndrome.

\section{REFERÊNCIAS}

Bernard G.R. \& Artigas A. 2016. The definition of ARDS revisited: 20 years later. Int. Care Med. 42(5):640-642.

Coimbra R., Junger W.G., Liu F.C., Loomis W.H. \& Hoyt D.B. 1995. Hypertonic/hyperoncotic fluids reverse prostaglandin E2 (PGE2)-induced T-cell suppression. Shock 45(4):45-49.

Davis H., Jensen T., Johnson A., Knowles P., Meyer R., Rucinsky R. \& Shafford H. 2013. Fluid therapy guidelines for dogs and cats. J. Am. Anim. Hosp. Assoc. 49(3):149-159.

Dellinger R.P., Levy M.M., Rhodes A., Annane D., Gerlach H., Opal S.M., Sevransky J.E., Sprung C.L., Douglas I.S., Jaeschke R., Osbom T.M., Nunnally M.E., Townsend S.R., Reinhart K., Kleinpell R.M., Angus D.C., Deutschman C.S., Machado F.R., Rubenfeld G.D., Webb S.A., Beale R.J., Jean-Louis V. \& Moreno R. 2013. Surviving sepsis campaign: international guidelines for management of severe sepsis and septic shock. Crit. Care Med. 41(2):580-637.

Durairaj L. \& Schmidt G.A. 2008. Fluid therapy in resuscitated sepsis: less is more. Chest 133(1):252-263.

Engen R.L. 2006. Dinâmica do sistema cardiovascular, p.163-172. In: Reece W.O. (Ed.), Dukes Fisiologia dos Animais Domésticos. 12ª ed. Guanabara Koogan, Rio de Janeiro.

Ferreira R.R., Barbosa P.R., Godinho E., Costa U.M., Gonzales F.H.D. \& Ferreiro L. 2004. Alterações hemato-bioquímicas em cães jovens com gastrenterite viral: relato de 18 casos. Medvep 7(2):159-163.

Friedman G., Soriano F.G. \& Rios E.C.S. 2008. Reposição de volume na sepse com solução salina hipertônica. Revta Bras. Ter. Intern. 20(3):267-277.

Garcia-Navarro C.E.K. 2005. Manual de Hematologia Veterinária. Varela, São Paulo.

Hauptman J.V., Walshaw R. \& Olivier N.B. 1997. Evaluation of the sensitivity and specificity of diagnostic criteria for sepsis in dogs. Vet. Surg. 26:393-397.

Jain N.C. 1993. Essentials of Veterinary Hematology. Lea and Febiger, Philadelphia.

Janeway C.A. 2007. O reconhecimento do antígeno, p.102-168. In: Janeway C.A. (Ed.), Imunologia: o sistema imune na saúde e na doença. 6aㅡ ed. Artmed, Porto Alegre.

Junger W.G., Liu F.C., Loomis W.H. \& Hoyt D.B. 1994. Hypertonic saline enhances cellular immune function. Circ. Shock 42(4):190-196.

Kaneko J.J. 1989. Clinical Biochemistry of Domestic Animals. 4th ed. Academic Press, San Diego.

Kogika M.M., Pereira D.A., Elias F., Notomi M.K., Delayte E.H., Kawahara R. \& Hagiwara M.K. 2003. Determinação sérica de haptoglobina, ceruloplasmina e glicoproteína ácida em cães com gastrenterite hemorrágica. Ciencia Rural 33(3):513-517.

Mendes R.S., Souza A.P., Silva R.M.N., Borges O.M.M., Torres L.M. \& Dantas A.K.F.P. 2011. Perfil hematológico e bioquímico de cães com gastroenterite hemorrágica por parvovírus diagnosticados pelo método de imunocromatografia. Acta Vet. Brasilica 5(3):278-283.

Mensack S. 2008. Fluid therapy: options and rational administration. Vet. Clin. Small Anim. 38(3):575-586.

Messick J. 2012. Sangue e medula óssea, p.61-78. In: Eurell J.A. \& Frappier B.L. (Eds), Histologia Veterinária de Dellmann. $6^{\mathrm{a}}$ ed. Manole, Barueri.

Rabelo R.C. 2013. Emergências em pequenos animais: condutas clínicas e cirúrgicas no paciente grave. Elsevier, São Paulo.

Serio A.B. \& Consorti L. 2013. Posição prona: por que e quando utilizá-la. In: Viana R.A.P.P. (Ed.), Sepse para Enfermeiros: as horas de ouro, identificando e cuidando do paciente séptico. $2^{\mathrm{a}}$ ed. Atheneu, São Paulo. 66p.

Silverstein D. \& Santoro-Beer K. 2013. Síndrome da resposta inflamatória sistêmica (SRIS), p.316-321. In: Rabelo R. (Ed.), Emergências de Pequenos Animais: condutas clínicas e cirúrgicas no paciente grave. Elsevier, Rio de Janeiro. 
Sorbo L., Ranieri V.M. \& Ferguson N.D. 2016. The Berlin definition met our needs: yes. Intern. Care Med. 42(5):643-647.

Strano T.R., Rocha S.L., Oliveira A.C.B., Bega D., Zammar G. \& Barbosa M.G. 2010. Análise comparativa da reposição volêmica com solução isotônica e solução hipertônica em ratos com sepse. Revta Bras. Ter. Intern. 22(4):321-326.

Tizard I.R. 2013. Innate immunity: macrophages and recovery from inflammation, p.41-51. In: Tizard I.R. (Ed.), Veterinary Immunology. $9^{\text {a }}$ ed. Elsevier, Missouri.
Unterer S., Busch K., Leipig W., Hermanns W., Wolf G., Straubinger R.K., Mueller R.S. \& Hartmann K. 2014. Endoscopically visualized lesions, histologic findings, and bacterial invasion in the gastrointestinal mucosa of dogs with acute hemorrhagic diarrhea syndrome. J. Vet. Intern. Med. 28:52-58.

Velasco I.T., Pontieri V., Rocha Jr S. \& Lopes A.U. 1980. Hyperosmotic NaCl and severe hemorrhagic shock. Am. J. Physiol. 239(5):664-673.

Ware L.B. \& Matthay M.A. 2000. The acute respiratory distress syndrome. N. Engl. J. Med. 341(4):1334-1349. 